\title{
The Light-Quantum Theory. ${ }^{1}$
}

\author{
By Dr. C. D. Ellis.
}

I $\mathrm{T}$ is well known that the wave-theory of light fails to explain certain phenomena, and so much has been written on this subject already, that it seems at first sight unnecessary to emphasise this point. But although the main problem has not altered and no solution has been found, yet the general attitude towards this question is slowly changing, and several important new experiments have been carried out. Recently a new theory was put forward by Bohr, Kramers and Slater which would have removed many of the difficulties, but experiments by Geiger and Bothe quickly showed it to be incorrect. This theory and its refutation were not without their effect, and now the attitude is frequently held that the light-quantum theory is not merely a crude picture of certain facts, but is an important theory no further from the truth than the wave-theory. The wave-theory itself cannot be correct, but except for its greater age it has no greater claims than the light-quantum view.

These two theories give entirely different pictures of the nature of light, as is expressed by their names. The wave-theory pictures the radiant energy as spreading out in all directions from the source, filling every portion of the surrounding space, so that the propagation may be imagined to be a handing on of the energy from point to point. It is an essential feature that except in special cases the intensity of the forces in the light wave diminish rapidly with increasing distance. Any theory will predict that the average energy density will diminish with the inverse square of the distance, but the law of variation of the forces is peculiar to each theory. The triumphs of the wave-theory scarcely need emphasis. By its means all the intricacies of interference and diffraction problems can be explained in the smallest detail, and in every problem involving direction this theory is in complete agreement with experiment.

It is only when we consider the interchange of energy between radiation and single atoms that the wave-theory breaks down, and the completeness of this breakdown is best seen by considering the photoelectric effect. To take a definite case, suppose X-rays are incident on a plate of some material, then it is found that electrons are ejected from the plate with considerable velocities. The number of electrons depends on the intensity of the X-rays and diminishes in the usual way as the plate is moved farther from the source of X-rays. The velocity or energy of each electron, however, does not vary but depends only on the frequency of the X-rays. The electrons are found to have the same energy whether the material from which they come is close to the X-ray bulb or whether it is removed away to any distance.

This is a result which is quite incompatible with the ordinary wave-theory of radiation, because as the distance from the source increases the radiation spreading out on all sides becomes weaker and weaker, the electric forces in the wave-front diminishing as the

1 Substance of three lectures on "The Atom of Light and the Atom of Electricity," delivered at the Royal Institution on February 25 and March 4 and $\mathrm{Ir}$.

NO. 2956, VOL. I I 7] inverse square of the distance. The experimental result that the photoelectron always picks up the same amount of energy from the radiation could only be accounted for by giving it the power either to collect energy from a large volume or to collect energy for a long time. Both of these assumptions are unworkable, and the only conclusion is that the radiated energy must be localised in small bundles.

This is the basis of the light-quantum theory. Light of frequency $v$ is considered to consist of small bundles or quanta of energy all identical and of magnitude $h v$, $h$ being Planck's constant. These quanta travel through space, being unaffected by each other, and preserving their own individuality until they make a suitable collision with an atom. Then if the quantum is absorbed the whole energy is transferred to the atom and an electron will be ejected. It can be seen that this hypothesis is so designed that the velocity of the photoelectrons is accounted for, first in the dependence on frequency, since the energy given up is always just one quantum, which bears the correct proportionality to the frequency; and secondly in its independence on the distance from the source, since although when
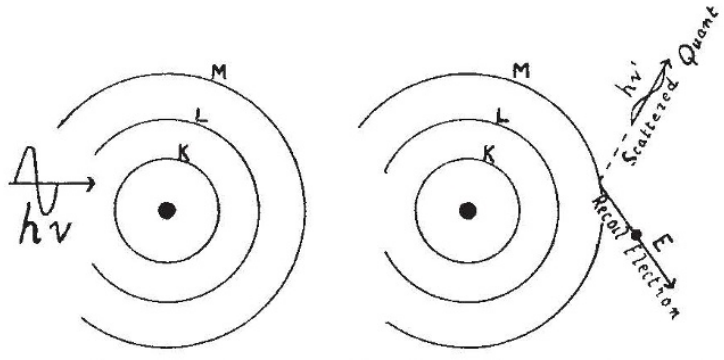

FIG. I.-Diagrammatic representation of the mechanism of the scatterin of X-rays in accordance with the relation $\mathrm{E}+h \nu^{\prime}=h \nu$.

the source is distant fewer quanta will arrive, those that do arrive will still give up the same invariable amount of energy to the atom.

This theory is also capable without further modification of accounting for the Compton effect. Compton found that $\mathrm{X}$-rays suffered a change of wave-length when they were scattered. Now, scattering on the light-quantum hypothesis means a deflexion of the quantum, and since this quantum with its energy $h v$ will have momentum $h v / c$ where $c$ is the velocity of light, this means further that the electron which deflects it will receive an impulsive blow. Fig. I, in which the orbital electrons of the atom are represented by concentric rings, illustrates such a process. The left-hand picture is supposed to represent the quantum just about to collide with an electron in the $M$ ring. The right-hand picture indicates how the quantum is deflected and sends off sideways the electron with which it collides. If, as the hypothesis demands, we consider energy to be conserved, then the energy of the scattered quantum plus the energy of the recoil electron must equal the energy of the initial quantum. In other words, the scattered quantum must have a smaller energy, which means lower frequency (since 
energy $=h v$ ) or longer wave-length. The theory at once suggests the simple qualitative result of Compton's experiments, and it is a striking point that the consideration of the simple dynamics of the collision lead directly to a complete quantitative agreement also.

It can be seen that the light-quantum theory explains two important phenomena, but in this crude form it fails to give any explanation of diffraction and interference just as completely as the wave-theory fails to explain the photoelectric effect. These two theories are clearly incompatible, and many attempts have been made so to modify either one or the other that it might explain the rival phenomenon. We find modifications of the wave-theory which attempt, on the whole unsuccessfully, to account for photoelectric phenomena; and again, there is another class of theories which make arbitrary rules to direct the light-quanta along such paths as would be necessary to give the correct interference patterns.

The most serious theory of the first class has been proposed by Bohr, Kramers and Slater, and it must be held greatly to the credit of this theory that it was sufficiently precise in its statements to be disproved definitely by experiment. The Bohr theory took its origin from the observation that it was only in the prediction and explanation of the energy relations that the wave-theory failed, and this failure could be briefly stated by saying that on the wave-theory there is never enough energy at the right place to produce the observed changes. These authors therefore suggested that energy might not necessarily be conserved in each interchange of energy between an atom and radiation, but only when a very large number of such interchanges were considered. For example, an atom which was in a radiation stream of frequency $\nu$ could suddenly acquire energy $h v$ by means of the action of the radiation, although the energy density of the radiation might be so small that this amount of energy could only be found in a volume a million times greater than that of the atom and there would be no possibility of imagining any kind of collecting process. However, if a very large number of such processes were considered, then the total amount of energy abstracted from the radiation would be equal to the energy absorbed hy the atoms.

The merit of this theory was that it rendered possible the calculation of the probabilities of such processes in a manner analogous to the calculations of absorption and emission on the pure wave-theory. It promised such a complete solution of the difficulty that immediately experiments were started to test its accuracy. One such experiment was carried out by Geiger and Bothe on the phenomenon of the Compton scattering, which has been referred to above. The essential features are that when $\mathrm{X}$-rays are scattered the scattered radiation shows an increased wave-length and low velocity electrons are liberated from the scattering atoms. The energy relations in this process are those that would be expected if a light-quantum endowed with mass and momentum were to collide with an electron and be deflected. As a result of the collision the electron will be driven sideways, forming one of the recoiling electrons. It is clear how the light-quantum theory predicts definitely that to every scattered quantum there must occur at the same moment a recoiling electron. On Bohr's theory these two events would not occur at the same moment, there would be no detailed connexion between any one recoiling electron and any particular portion of the scattered radiation. All that is required is that, on the average, energy should be conserved. From this it may be seen that a decision between the two theories will be reached if it is possible to settle whether to each recoiling electron there corresponds a scattered quantum, or whether the scattered radiation is radiated continuously.

Geiger and Bothe directed a stream of X-rays on to a small volume of hydrogen, and on either side of this were arranged two counters. These consisted of small chambers charged to a high potential with an inner insulated electrode. When a single electron traverses the gas in such a counter there is a sudden flow of electricity to the electrode, which may be registered by a suitable instrument. One counter was open to receive the recoiling electrons, the other was to register the radiation, and was closed by a thin metal foil. On the light-quantum view we should say that the quanta incident on the foil will sometimes eject an electron into the counter, which will then register it. Since every recoiling electron must be accompanied by a scattered light-quantum there should be a detectable number of coincidences between the indications of the two counters. On Bohr's theory the scattered radiation comes off in a continuous stream, and passing through the metal foil on the face of the counter, will from time to time liberate electrons. Although on this theory there should be just as many electrons liberated as on the previous theory, the important point is that now the secondary electrons will have no direct connexion with the recoiling electrons, and there would be no reason to expect coincidences between the indications of the two instruments.

The result of this experiment was decisive; undoubted coincidences were found to occur between the indications of the electron counter and the radiation counter. This could not be accounted for on Bohr's theory, which consequently had to be abandoned. But at the same time it provided additional support for the light-quantum view, although it certainly is not a proof of its correctness.

Bothe has recently carried out a further experiment on similar lines which is specially interesting, since in this case Bohr's theory would predict coincidences whereas the light-quantum theory would not. A small piece of iron foil was placed between two ' radiation' counters and radiated with $\mathrm{X}$-rays so that it emitted its own characteristic radiation. If this radiation consisted of localised quanta, then it is clear that any one quantum could go into either one counter or the other, but it could not possibly be absorbed in both counters at the same time, and there should be no coincidences between the indications of the two counters. On the other hand, if the characteristic radiation from the foil spreads out in a spherical wave, this wave will pass through both counters at the same time, and occasionally will stimulate an absorption act in both counters simultaneously, so that on this theory coincidences should be found. Experiment again 
decides definitely against the theory of Bohr, Kramers, and Slater, and gives the result that would be expected on the light-quantum view. This is the fate of the most serious theory which has attempted to solve the fundamental difficulty by preserving the wave-theory but altering our pictures of the absorption and emission of energy by atoms.

There is another group of theories which postulate that radiation consists of discrete quanta. Thus they explain automatically the photoelectric effect and the Compton scattering, and then attempt to account for diffraction and interference by laying down rules which shall determine the paths these quanta shall follow. The light-quantum theory accounts for all the effects once the quanta are got to the right place, but in its crude form it gives no suggestion of the quanta moving in anything but straight lines, and it is only the wave-theory which predicts successfully the directions in which light travels. Hence theories have been proposed which provide 'tramlines' along which the quanta move, and these 'tramlines' are so arranged that in a system of interference bands "tramlines ' only lead to the bright bands and none to the places of zero intensity. To be more precise, the 'tramlines' are supposed to be the paths of the Poynting vector of a virtual radiation field emitted by the atoms, and the probability of a quantum travelling along a given path is connected with the value of the Poynting vector along that path. Such theories meet with many difficulties, and no one has yet been carried to the point where it can be tested by experiment.

This has been discussed in detail recently by Prof. G. N. Lewis in an article in NATURE (February I3, p. 236 ), in which he also puts forward an original view on the whole problem. It is a striking fact that while all the theories are directed towards explaining the propagation of light, one theory suggesting that it occurs in the form of waves, the other in the form of corpuscles, yet light has never been observed in empty space. It is quite impossible to observe light in the course of propagation; the only events that can ever be detected are the emission and absorption of light. Until there is some atom to absorb the radiation we must be unaware of its existence. In other words, the difficulty of explaining the propagation of light may be because we are endeavouring to explain something about which we have no experimental evidence. It might be more correct to interpret the experimental facts quite directly and to say that one atom can transfer energy to another atom although they may be far apart, in a manner analogous to the transference of energy between two atoms which collide. Prof. Lewis says: "I shall make the contrary assumption that an atom never emits light except to another atom, and that in this process, which may rather be called a transmission than an emission, the atom which loses energy and the atom which gains energy play co-ordinate and symmetrical parts."

On this view the fringes in an interference system are to be regarded as forming one system with the source in the sense that the atoms which absorb the light at the bright fringes are in 'virtual ' contact with the atoms in the source, whereas those in the dark fringes or any place where the light does not go are not in virtual contact. On this view there would never be any discussion as to where radiation goes, but only as to which pair of atoms could make up an emitting and absorbing couple.

The foregoing shows clearly that there is at present nothing approaching a complete theory of light, but yet it must be remembered that it is an important practical achievement that a complete description can be given by means of two theories, providing the appropriate theory is used for each phenomenon. Both these theories have more than justified the care and trouble spent on their elaboration by the help they have given to experimental work, and also on the theoretical side they represent an important advance in our knowledge of light. It would not be right to deduce that both theories are necessarily incorrect; it might be closer to the truth if we were to compare them to the plan and elevation of an engineering drawing. The wave-theory and the quantum-theory may be just the plan and elevation of the true theory of light, and the marked contradictions between them may be due to the language we use to describe the experimental results, just as the differences between a plan and elevation reflect our attempt to describe in two dimensions a three-dimensional object.

\section{The Origin of Petroleum.}

\section{By Henry B. Milner.}

E VERY branch of natural science possesses its quota of time-honoured problems awaiting solution ; these serve to maintain an essential stimulus to contemporary thought and to animate that discussion from which progress alone is possible. Controversy, however, unless confined within legitimate bounds of clear issues, is prone to irrelevancies and digressions to an extent that, proverbially, the 'forest ' of significant observations tends to be blurred by the 'trees' of individual conjecture and detail: thus is truth obscured and explanation postponed. So it is with the science of petroleum; the problem of its genesis remains paramount, partly because there are more hypotheses than even plausible solutions based on fact, mainly for the reason that the real perspective of the problem is being lost in the maze of accumulated detail and argument. It was not only appropriate but essential that, to avoid further confusion, some one should see round rather than into the problem of mineral oil origin, that the ground should be cleared by correlation of definite data of constructive theories, thus propounding a unified hypothesis from which future research could trend with some hope of positive results. It is from this viewpoint that Mr. Ernest Clark has surveyed his subject, and his thesis (presented to the Institution of Petroleum Technologists on May 25) more than justifies his entry into this difficult field of debate, chiefly by his careful analysis of the situation, by his elimination of the unessential, and by his attempt (entirely successful) to point the way. 